Théologiques

Théologiques

\title{
Théologie de la création : une nouvelle pertinence
}

\section{Pierre Gisel}

Volume 2, numéro 1, mars 1994

Création et créativité

URI : https://id.erudit.org/iderudit/602396ar

DOI : https://doi.org/10.7202/602396ar

Aller au sommaire du numéro

\section{Éditeur(s)}

Faculté de théologie de l'Université de Montréal

\section{ISSN}

1188-7109 (imprimé)

1492-1413 (numérique)

Découvrir la revue

\section{Citer cet article}

Gisel, P. (1994). Théologie de la création : une nouvelle pertinence.

Théologiques, 2(1), 25-43. https://doi.org/10.7202/602396ar

\section{Résumé de l'article}

La modernité occidentale, aux visages multiples, a occasionné des déplacements quant à la position et au rôle de l'humain sur la scène du monde. L'anthropocentrisme (de même que le biocentrisme) est au centre d'un procès qui ne doit prôner ni le retour ni la résorption à un ordre naturel. Cet article veut proposer une voie qui fasse écho au thème de la création en lien avec la tradition biblique et chrétienne bien comprise. Cette voie accepte la contingence du monde et la transcendance de Dieu, et reconnaît que les différences entre savoir et croire ne mènent pas à la séparation des deux domaines. 
Théologiques 2/1 (1994) 25-43.

\title{
Théologie de la création : une nouvelle pertinence
}

\author{
Pierre GISEL \\ Faculté de théologie \\ Université de Lausanne
}

\section{RÉSUMÉ}

La modernité occidentale, aux visages multiples, a occasionné des déplacements quant à la position et au rôle de l'humain sur la scène du monde. L'anthropocentrisme (de même que le biocentrisme) est au centre d'un procès qui ne doit prôner ni le retour ni la résorption à un ordre naturel. Cet article veut proposer une voie qui fasse écho au thème de la création en lien avec la tradition biblique et chrétienne bien comprise. Cette voie accepte la contingence du monde et la transcendance de Dieu, et reconnait que les différences entre savoir et croire ne mènent pas à la séparation des deux domaines.

\section{Prélude. La modernité: un paradigme mis en cause}

La modernité occidentale est née d'une révolution dans le rapport de l'homme à la nature et au cosmos. Fin d'un monde tout à la fois foncièrement unitaire et différencié, fait d'analogies multiples, de correspondances et de renvois possibles, metrant tout particulièrement en consonance l'univers lui-même (le macrocosme) et l'être humain (le microcosme); début d'une odyssée qui voit l'homme délogé de toute position centrale, obligé d'assumer sa place, son histoire et son identité sur fond de fragilité et d'énigme. Début d'une aventure.

Une matrice biblique d'origine juive déployait peut-être là ses effets, mais non sans une radicalisation et une autonomisation de certains de ses éléments. Un jeu de différences constitutives entre Dieu, le monde et l'homme faisait en effet place à découplage et rupture. La gloire de l'être 
humain se montrait dès lors dans la force manifestée à faire "table rase», à investir de nouveaux commencements et à donner forme à son environnement naturel et social.

On a pu dire de cet être humain qu'il accédait à l'âge adulte; d'autres l'ont vu sourdement orphelin. On a pu chanter sa liberté; d'autres l'ont soupçonné d'être prométhéen. Quoi qu'il en soit, la modernité était née. Règne de la raison et parfois de la technique, d'une instrumentalisation ou d'un fonctionnalisme envahissant. Règne du politique, pouvant laisser sourdre l'absolutisme ou le totalitarisme. Règne des singularités individuelles, le plus souvent en mal de médiations avec les autres, le monde, Dieu ou les dieux, et finalement avec soi-même; en mal de mémoire et de projet aussi, et dès lors d'institutions articulées, tout à la fois reconnues et aménageables.

Un tel changement de décor n'a pas laissé indemne la théologie (ni la foi!) chrétienne. Le discours sur le monde et la nature s'est fait plus discret, au profit d'un regard plus délibérément centré sur les réalités humaines, psychologiques et sociales. On a cherché à repenser la pertinence de la théologie, d'une façon qu'on voulait plus limitée et plus spécifique. C'est ainsi que la théologie moderne a souvent favorisé une déconnection des questions liées aux savoirs sur le monde et celles touchant le croire. Le protestantisme me semble avoir illustré tout particulièrement ces modifications de conjoncture, ne serait-ce qu'en les assumant; mais le catholicisme n'y fut pas indifférent non plus, comme peut notamment l'attester un regard plus délibérément centré sur les réalités ecclésiologiques qui s'est fait alors jour ${ }^{1}$. En profondeur, une perspective marquée par les thèmes d'une théologie de la création laissa passer à l'avant-scène une vision commandée par les motifs d'une théologie du salut (ou de la "désaliénation", voire de la "libération"), dont les formes furent diverses, allant même jusqu'aux expressions sécularisées qu'en constitue à mon sens un primat accordé aux problématiques morales ${ }^{2}$.

Nous héritons aujourd'hui de cette modernité. Tant les données qui furent les siennes que son projet même balisent pour une part la

1 Jusqu'à se vouloir, souvent, "contre-société» (ce qu'il est actuellement convenu d'appeler le «catholicisme intransigeant»).

2 Ce primat est typique d'un certain «néo-protestantisme», en particulier autour d'Albrecht Ritschl (1822-1889), et d'une certaine manière d'en appeler à Kant. On peut estimer que nous vivons aujourd'hui, sur certains points, une situation analogue. 
conjoncture dans laquelle nous vivons et que nous avons à prendre en charge. Pourtant, en même temps, chacun le pressent, et tout particulièrement à propos du thème qui nous retient ici, le paradigme classique de notre modernité occidentale est en crise. L'illustrent tour à tour la conscience écologique protestant contre une réduction de la nature à un objet d'exploitation et de maîtrise, les impuissances ou les dangers d'un projet socio-politique organisé autour de la seule raison et d'une vision globalisante, les craintes ou les rejets divers qu'engendre le recours à une technique et à un fonctionnalisme unidimensionnels. Nous vivons le temps où ce qui fut réprimé fait retour. D'une façon qui peut apparaître irrationnelle ou "sauvage" parfois; mais qui en appelle à d'autres approches ou à d'autres logiques. C'est en fonction de cette conjoncture globale me semble-t-il que nous sommes aujourd'hui aux prises avec une interrogation nouvelle et urgente touchant nos institutions, les médiations qu'elles mettent en place et dont elles vivent, les rapports à l'autre et à nos identités qu'elles régulent, avec ce qui s'y profilent de questions touchant nos liens avec notre passé (nos généalogies et notre mémoire), avec le cosmos (ce qui tout à la fois nous précède, nous nourrit et nous provoque), avec les représentations que nous nous donnons ou dans lesquelles nous nous reconnaissons (nos expressions religieuses et culturelles, nos valeurs et nos dieux).

À mes yeux, une telle donnée présente des dimensions techniques, mais politiques aussi, psychologiques, culturelles et religieuses. Dimensions tout à la fois irréductibles et en interférences complexes. Dans les pages qui viennent, je me situerai comme théologien, et de tradition chrétienne; la dimension mise en avant sera donc «religieuse», mais elle le sera au gré d'une mise en perspective historique et réflexive propre, orientée. Sa validité est celle d'une exemplarité parmi d'autres possibles (à ce titre, elle devrait s'exposer à un dialogue interreligieux et s'en trouver tout à la fois reformulée et déplacée); s'y joint un intérêt complémentaire, de ce que la matrice religieuse en cause, biblique et chrétienne, et notre histoire occidentale furent de fait liées. En ce sens, la mise en perspective historique et réflexive proposée vaudra aussi, à sa manière, comme apport à une généalogie globale de notre donné occidental présent.

\section{Entre le procès de l'anthropocentrisme et le retour à la nature}

L'appel à un retour à la nature se donne aujourd'hui libre cours. Il prend des formes diverses et pas toujours conciliables. On peut évoquer - c'est l'un des cas les plus nets - le mouvement de la Deep Ecology 
(visant une osmose entre le soi et la nature, à l'enseigne d'un biocentrisme ${ }^{3}$ ), les multiples tentatives de prendre en charge sur mode alternatif tant les questions de la santé que de l'alimentation (pouvant évoquer la nature, la tradition ou telles forces cosmiques, en rupture avec le savoir médical autorisé et ses techniques), les développements de psychologies intégratives (plus «holistiques» ou plus "positives» que les modèles d'obédience freudienne valorisant le travail de deuil et l'accession d'une singularité finie à elle-même), la mise avant de valeurs plus unifiantes peut-être tout particulièrement portées par les femmes (contre les thèmes de l'altérité et les faits de domination d'une ère "patriarcale»), les développements, en Occident, européen ou américain, de mouvements religieux prônant d'une manière ou d'une autre un rapport décisif au cosmos (et à ce titre en rupture avec les formes judéochrétiennes des Églises instituées), un nouveau rapport à la mort (et donc à la vie), qui tend désormais plus à être vue comme "passage" (naturel...) que comme énigme ou tragique, à la source de révoltes, de protestations ou de sublimations diverses. On peut y ajouter, pour le moins comme confirmation indirecte d'un déplacement des problématiques ou des interrogations, un regain d'intérêt pour la nature dans les champs philosophique, éthique et théologique 4 .

Derrière ces différents mouvements et ces quêtes nouvelles se profile un déplacement net. L'humain quitte la position centrale qu'il occupait sur la scène du monde. Sa différence propre, singulière er irréductible, tend à n'être plus validée comme telle. Ni l'aventure qui peut s'y articuler. Ni donc l'histoire et la liberté qui s'y inscrivent ou qui l'instituent. Dès lors, c'est en fonction de l'ordre cosmique et de la nature qu'il est invité à être. L'homme réintègre un donné foncièrement unitaire, aux résonances multiples et harmonisées. On peut parler de destin, d'appartenance, d'approfondissement, de répétition essentielle (de mimesis), voire de

3 Voir Les sentiments de la nature. (BOURG, Dominique, éd.), Paris, La Découverte, 1993. Sur l'ensemble de la problématique en jeu, on pourra lire aussi La nature en politique ou l'enjeu philosophique de l'écologie. (BOURG, Dominique, éd.), Paris, L'Harmattan, 1993, et Religion et écologie. (HERVIEU-LÉGER, Danielle, éd.), Paris, Cerf, 1993.

4 Voir Éthique et natures. (FUCHS, Eric et HUNYADI, Mark, éd.), Genève, Labor et Fides, 1992; De la nature. Paris, Beauchesne, 1992; «Nature et culture», Le Supplément 182-183 (1992); la réception de la pensée de Hans JONAS (Le Principe responsabilité. Paris, Cerf, 1990) et les débats qu'il a suscités (voir Esprit 140-141, 1988, et 171, 1991; Nature et descendance. Genève, Labor et Fides, 1993; Aux fondements d'une éthique contemporaine. (HOTTOIS, G., éd.), Paris, Vrin, 1993). 
retour à l'origine. Les dieux changent également. On quitte le Dieu d'une différence absolue, instituant ex nihilo tant l'homme que le créé (euxmêmes en différence et dès lors foncièrement décentrés) au profit de dieux qui ont foncièrement à voir avec la vie et ses jeux infinis.

Qu'en penser? Le procès d'un anthropocentrisme foncier, lové au coeur de l'aventure occidentale de notre modernité classique, me paraît tout à la fois légitime et requis; Nietzsche l'avait d'ailleurs déjà instruit à sa manière, y diagnostiquant des traces d'auto-idolâtrie, de réduction et de peur, d'incapacité à faire face à l'autre, au monde, à la luxuriance du réel, voire à l'énigme, en tout cas à la gratuité et au destin. Le théologien ne peut en effet que se montrer critique à l'égard d'une certaine figure de la modernité, qui fut à bien des égards socialement et techniquement dominante. Mais à cela, il faut immédiatement ajouter que cette figure moderne naît d'une aspiration plus complexe, qu'il s'agit aussi - tout autant même, à mes yeux en tout cas - d'honorer et donc de savoir déchiffrer. Théologiquement - et cela dit non sans anticiper tel ou tel élément d'une position de fond - la modernité ne doit donc pas être critiquée au nom et au profit d'une vision passéiste, pré-moderne. On évitera ainsi par exemple - surtout en un ordre ecclésial qui peut éprouver là trop facilement sa tentation propre - de «diaboliser" la liberté au profit des appartenances données, ou la raison au profit des traditions ou des autorités reçues, ou encore l'avènement des singularités humaines au profit d'une révélation passée et qui serait suffisante en son donné même ${ }^{5}$.

Un anthropocentrisme proprement moderne doit assurément être dénoncé, mais non sans, peut-être, une lecture plus différenciée de sa cristallisation effective, ainsi que de ce dont il procède - qui est complexe - et de ce dont à sa manière - certes malheureuse - il témoigne et qui pourrait être repris, autrement justement. Historiquement et culturellement d'ailleurs, je dirais volontiers qu'à tout prendre, à un regard théologique, la modernité anthropocentrique apparaît certes comme l'enfant d'une matrice judéo-chrétienne, mais comme un enfant bâtard. Il s'agit dès lors assurément de s'expliquer sans complaisance avec cette généalogie de fait, mais tout à la fois de discerner, critiquement et pour

5 Rappelons qu'il n'y a pas, en perspective chrétienne bien comprise, de «révélation» sans un incognito central (marqué par une non-reconnaissance et la croix) et dès lors sans le jeu d'un renwoi (indirect) au Père: un jeu "dans l'Esprit» (sur ce point, voir mon petit livre Corps et esprit. Genève, Labor et Fides, 1992). 
soi, en quoi se sont aussi marquées là des discontinuités ou des déviations, et donc en quoi peuvent se profiler des correctifs possibles.

Ajoutons que la modernité occidentale classique - je l'ai laissé entendre - n'offre pas un visage uniforme. Sa figure socialement dominante et actuellement objet de procès fut, tout au long, accompagnée d'une veine différente qui est elle aussi moderne à sa manière (elle fait le plus souvent couple avec la première): une note protestataire contre la réduction à une raison analytique, l'appel à une multidimensionalité qui ne va pas sans résonner avec le religieux, l'histoire ou la tradition, la vision d'une socialité intégrée ainsi que l'engagement en vue d'une expression esthétique autre que compensatoire. Sa figure classique est celle du romantisme, allemand notamment, né à l'ombre des "Lumières européennes" justement. Ses relais, divers, ont ponctué notre histoire tout au long du XIX siècle et du XXe $\mathrm{X}^{\mathrm{e}}$. Or, classiquement, le romantisme fut déjà à mon sens, en profondeur, l'affirmation d'une nature homogène, invitant l'homme à y trouver sa place plus sur le mode d'une participation foncière, voire d'une similitude cachée à redécouvrir, que d'une différence à marquer ou à instituer, vivant plus de nostalgie que de témoignage rendu à l'altérité, visant plus d'épousailles que n'en appelant à un désir en excès et à jamais inassouvi 6 .

Procès de l'anthropocentrisme? Certes. Pour le bien de l'humain, individuel et social. Et pour la survie de tous. Parce que l'homme n'est ni l'origine ni le centre de toutes choses. Parce qu'il doit être foncièrement à l'écoute et en situation d'accueil. Parce qu'il est corps. Prêt à être subverti et appelé à se recevoir. Mais si le procès est légitime et requis, il doit éviter de donner prise au ressentiment. Il doit être conduit de façon différenciée, ce qui évitera qu'on ne soit entraîné à passer d'un extrême à l'autre. En matière personnelle aussi bien qu'historique, un pur et simple renversement s'avère trop dépendant de ce qu'il refuse pour être vraiment fructueux; personnellement et collectivement, on ne vit d'ailleurs pas de pure rupture, mais bien des différenciations qui nous permettent d'exister, sachant d'où nous venons (ce qui nous situe et nous porte) et aptes à nous exprimer en notre nom propre (singulier et critique).

Procès de l'anthropocentrisme? Oui. Mais non pour chanter un retour ou une résorption à un ordre naturel. Entre ces deux termes extrêmes, il y a place pour la réflexion et la construction d'une troisième voie,

6 Sur ce point, voir ma contribution «Pertinence du religieux et instance théologique» (Mélanges Christian Duquoc), Paris, Cerf (à paraître). 
différente. Personnellement, elle me paraît seule susceptible de donner écho à la tradition biblique et chrétienne bien comprise, relue certes à la lumière de notre histoire effective (de ses aspirations et de ses impasses) et repensée pour notre aujourd'hui culturel, social et personnel, intégrant donc - comme il se doit! - une ou des quêtes propres à notre temps (elle aura en ce sens-là, nécessairement et légitimement, figure moderne!).

\section{Configuration d'une théologie biblique et chrétienne de la création}

Notre rapport à l'originaire est, je crois, décisif. Pour tout homme et toute femme. Il l'est dans notre vie individuelle et collective. Il peut être explicité dans des mythes, des images ou des phénomènes d'identification complexes; il peut être implicite. Il se love quoi qu'il en soit au coeur de notre rapport au monde, à autrui, à soi. En outre et à l'évidence, les civilisations et les cultures, de même que nos itinéraires plus personnels, en mettent en avant des représentations diverses. Comme sont diverses nos manières d'habiter le monde présent, de dire nos identités, notre articulation à un passé ou nos projets possibles.

La perspective biblique et chrétienne sur la création présente, comme d'autres, une configuration particulière en la matière. Différente d'autres; émargeant peut-être aussi à un éventail finalement non infini de postures humaines où peuvent se cristalliser, dans la différence justement, une même quête ou des besoins semblables, un jeu analogue entre le destin, le désir, l'acceptation, le dépassement ou la résolution, et cela sur des registres également analogues que strient les échanges du multiple et de l'un, de l'altérité et de l'identique, de l'être et de son au-delà (par négation ou transmutation), de la vie et de la mort, de la contingence et des nécessités, ou encore du corps et de l'esprit.

Les mythes - avoués comme rels ou non, avec leurs possibles régulations religieuses ou autres - ont leur importance. Irréductible, même si ne s'y joue qu'une des instances qui président au déploiement concret de la vie humaine. Il est absurde à mon sens de vouloir harmoniser ce qui s'y dit et le savoir scientifique. Cela vaut pour la configuration biblique et chrétienne comme pour celles d'autres sphères culturelles et religieuses. Prétendre le contraire ne pourrait que nous conduire - sauf coup de force autoritaire en faveur d'une représentation au détriment des autres - à un dénominateur commun à la fois trop restreint et trop général, et du coup trop abstrait pour être signifiant. La pluralité est par ailleurs source d'enrichissement et ne peut, indirectement mais réellement, 
que nous provoquer à un approfondissement salutaire de ce qui est chaque fois en jeu, dans nos propres représentations pour commencer.

L'harmonisation entre représentations religieuses d'une part d'expression mythique et d'adhésion croyante - , et savoirs scientifiques de l'autre est au surplus vouée à l'échec, comme le montrent tous les efforts «concordistes» qui ont traversé l'époque moderne. Les savoirs scientifiques changent en effet. Le constater n'en invalide pas la pertinence et la vérité, concourt tout au plus à en préciser les limites - internes - répondant, en leur ordre, à des limites analogues quant à l'ordre de pertinence et à la vérité possible des mythes et des représentations religieuses.

Le refus de l'harmonisation me paraît enfin commandé, de manière plus spécifique, par une juste compréhension de la tradition biblique et chrétienne. Le hiatus entre savoirs et confession, l'énigme qui s'y loge et l'irréductibilité d'instances qui s'ensuit, me semblent en effet requis par le propos le plus central du donné biblique et chrétien sur la création. Esquissons en quoi et comment.

Les traits constitutifs de la confession biblique et chrétienne relative à l'originaire peuvent être rappelés brièvement, même si j'en crois la richesse grande et susceptible d'alimenter toujours à nouveau une méditation pouvant conduire à approfondissement et à (re)découverte ${ }^{7}$. Il suffit d'évoquer ici les deux récits de commencement situés au départ du texte canonique chrétien, Genèse 1 et Genèse 2-3. On y lit que, pour l'humain et pour la vie dans la création, la différence est originaire et constitutive. Différence dans l'ordre même de ce qui est créé et qui vaut comme monde: différences spatiales (haut/bas; sec/humide; etc.), temporelles (jour/nuit; commencement structuré selon l'ordre total et déployé d'une semaine inaugurale; etc.), biologiques (végétal/animal; espèces diverses), anthropologiques (homme/femme). Et différence touchant ce sur le fond de quoi advient ce créé ou ce monde: pas de lien continu ou homogène du Créateur aux créatures (sur mode d'engendrement ou d'émanation par exemple), mais une institution ou une irruption sur mode d'altérité, conduisant notamment à tenir que le monde n'est, rigoureusement, en rien divin. Il y a certes «alliance» possible et trace de "correspondance" - l'humain, homme et femme, est créé «à l'image de

7 J'ai eu plusieurs fois l'occasion de le développer, notamment dans «Nature et création selon la perspective chrétienne», dans Religion et écologie, p. 29-45; «La création. Thèses théologiques», CahProt 5 (1988) 30-36; "Création. Méditation théologique», dans Dieu, le monde et l'homme. Hasard ou projet?. Paris, O.E.I.L., 1988, p. 158-169, et bien sûr mon ouvrage La création. Genève, Labor et Fides, 1980, 19872 
Dieu" - , mais ce sera par delà une différence et une séparation, sur le fond de cette différence, tout à la fois permise et provoquée par elle. Le créé peut - doit! - faire l'objet d'une reprise - en écho - et être ainsi le lieu d'une résolution, mais la reprise ou la résolution sera interne; le créé peut être l'objet d'une offrande, dans un cadre donné et selon une perspective orientée, mais ce sera une offrande en différence. L'altérité Dieu/humain s'en trouve médiatisée par le rapport incontournable, décisif et seul prometteur, par le monde, par son épaisseur, sa différence propre et les énigmes qui s'y condensent; l'écho donné à l'originaire, sous forme de reprise ou d'offrande, sera donc indirect.

J'ai jusqu'ici fait résonner, sommairement, le texte de Genèse 1. Le récit de Genèse 2-3 - dont le système de représentation cosmologique est tout différent, notons-le au passage - vit en profondeur, à mon sens, du même type de rapport à l'originaire. Le récit est centré sur l'histoire de l'humain dans la création en son acte le plus central, où se décide qui est cet humain. Rappelons d'abord que l'intrigue se déploie et se noue sur le fond d'un espace éminemment relationnel (l'homme en rapport à la terre, aux animaux, à l'autre sexe), au coeur d'un monde qui, loin d'être la plage vierge d'un commencement premier et absolu, a dès le départ figure de "jardin", cultivé et à cultiver (l'humain y est placé, de l'extérieur) et qui présente de surcroît une figure éminemment structurée comme l'atteste de façon hautement symbolique l'arbre central, pour une part arbre-de-vie (donc de mort possible) et arbre-limite (donc de transgression possible).

Par delà une telle mise en scène, l'enjeu au plus profond de l'intrigue elle-même me paraît des plus significatifs. Il confirme et porte à son incandescence tout ce qui a été rapidement esquissé jusqu'ici. Parce qu'il est inscrit en différence et non sur fond d'homogénéité, parce qu'il est séparé de Dieu, donc hors toute dépendance directe et linéaire, l'humain est moins assuré dans l'être qu'il n'est appelé à recevoir la création et à en répondre: appelé à être. Bibliquement et selon la théologie chrétienne, l'existence personnelle, décidée en responsabilité singulière, fait ainsi partie intrinsèque de l'être. On est ici dans l'ordre du nom, de la précédence spécifique et de la structure d'interpellation auxquelles il est lié, ainsi que dans l'ordre de l'histoire, de la vocation propre et de la structure généalogique qui la commandent. Et pour l'humain, tout se décide «originairement» au coeur du temps (de toujours dejà commencé) et de la création justement (de toujours dejà donnée); tout se décide "originairement" au coeur des choses et aux prises avec elles. "Originairement", c'est-à-dire: radicalement ou à la racine. Mais «originairement» à bon droit, dans la mesure où cette décision radicale l'enjeu inscrit au coeur de l'intrigue ou de la dramatique mise en place - 
se noue précisément en fonction d'une question portant sur le commencement. La tentation placée dans la bouche du serpent et liée à l'arbre-de-vie/arbre-limite dressé au coeur du jardin le manifeste: "vous serez comme des dieux". Tel est en effet l'enjeu: vivre de la création déjà donnée, à partir d'elle et en se sachant donc n'être pas soi-même à l'origine (ni être soi-même origine), ou se laisser prendre à son propre mirage, vivre a partir de soi, hors la création donnée, dans l'illusion de la posséder dans le savoir et la maîtrise, et être ainsi, rigoureusement justement, "comme des dieux». D'un côté, une finitude reconnue peut s'insérer dans la vie et être porteuse de vie; de l'autre, une dénégation et un rêve d'infini m'inscrivent dans la mort et me font porteur de mort: le mensonge dans lequel s'installent Adam et Ève, dans leur relation à euxmêmes et à Dieu; la violence ou la non-reconnaissance tout à la fois de l'énigme (le sacrifice refusé) et du frère (supprimé) en laquelle s'enferme Caïn.

La dramatique est ici celle d'une liberté - non d'une obéissance hétéronome -, mais d'une liberté située; celle de la figure humaine comme telle - non d'un particularisme - , mais de la figure humaine inscrite dans une condition, tout à la fois non choisie et concrète; celle d'un accomplissement - non d'un état de chose en lequel se fondre ou sur lequel s'assurer -, mais d'un accomplissement dont la réalisation doit prendre corps au plus intime du réel, aux prises avec lui (le réel est-il visage de destin ou occasion de bénédiction?), confronté à ses énigmes et à son opacité (d'où vient-il?); celle d'une histoire enfin - non d'un retour à un premier commencement ni une résorption dans les choses -, mais d'une histoire où se noue un geste qui porte le monde plus avant en direction de ce qui le dépasse: le réel est pour ainsi dire mystérieusement recueilli en l'homme (le réel "cultivé" ou à réoffrir «devant Dieu») dans le moment même où il est reçu comme venant d'en deçà de lui-même (il vient de Dieu, autre et hors monde).

La méditation du mythe ici en cause fait apparaître encore un point qui confirme ou en quelque sorte radicalise le propos, j'entends le dédoublement qu'induit, en termes de commencement, la non-continuité, la non-linéarité ou l'hétérogénéité sur le fond de quoi la dramatique originaire - "adamique» - est racontée. Il y a en effet la précédence bonne de la création, irrésorbable en contemporanéité (l'humain n'advient qu'apres et au coeur du créé); et il y a la précédence du mal (symbolisé par la présence du serpent au coeur d'une création bénie et dite bonne), précédence tout aussi irrésorbable: elle passe l'homme ou l'excède, si l'homme est sujet du mal, en toute responsabilité, il ne l'«invente" pas, mais y répond. 
Que ce soit par rapport au réel, donné et rapporté à un Dieu hors monde, ou que ce soit par rapport au mal, en excès tout en venant s'inscrire au plus intime de mon histoire (un mal qui naît et renaît du fantasme qui me voudrait hors-lieu, hors-temps et donc hors-monde), l'humain n'existe ainsi qu'en généalogie: appelé à naître à lui-même par delà une différence originaire, au gré de cette différence même et tout en la dépassant sans la résorber; se situant ainsi face à ce qu'il n'est pas mais dont indirectement il vient, appelé à faire mémoire d'un «immémorial» inscrit dans et par delà son rapport au passé et à lui-même, et voué à un "inespéré" inscrit dans et par delà son rapport au futur et à lui-même.

Enfin, que la dramatique «adamique» ici mise en scène soit fondamentale dans la perspective biblique et globalement chrétienne ${ }^{8}$, l'atteste la mise en scène du Christ Jésus - «nouvel Adam" selon l'appellation profondément théologique de l'apôtre Paul - dont les évangiles racontent en prélude à son ministère terrestre le moment originaire, constitutif à certains égards, dans la logique du récit tout au moins. On y voit Jésus au désert, et objet de tentation. Une tentation triple, mais même à chaque fois. Surtout, c'est là, en profondeur et théologiquement, rigoureusement la même tentation que celle d'Adam: tentation d'infinitude, croyant pouvoir faire l'économie des limites de la création (pour le bien!), et être ainsi roi ou pour le moins reconnu: «fais de ces pierres du pain, et tous mangeront». Ou, ce qui revient au même et fait apparaître l'illusion et le mensonge: «saute du haut du temple, les anges te porteront et du coup les foules te suivront"; ou encore: "adoremoi, et le monde sera à toi".

\section{L'humain entre contingence (donnée) et absolu (hors prise)}

J'ai jusqu'ici balisé des héritages et des enjeux (liés à notre situation historique propre), fait état de tel ou tel refus à l'endroit de tentations qui nous sollicitent aujourd'hui (tant d'un anthropocentrisme que d'un biocentrisme) et donné forme à une relecture du motif biblique et chrétien touchant le rapport de l'humain et de la création, une relecture conduite en lien avec une interrogation généalogique de notre histoire et avec des problématiques lovées au coeur de notre présent. Une orientation d'ensemble s'en dégage, qui se veut tout à la fois proposition assumable

8 Pour une perspective spécifiquement juive (non sans profond parallèle à mon sens), voir Catherine CHALLIER, «L'alliance avec la nature selon la tradition hébraïque», dans Religion et écologie, p. 17-28. 
en responsabilité personnelle et relève possible des questions contemporaines qui requièrent chacun. Il reste à en esquisser quelques conséquences, à ouvrir certaines pistes. Elles concerneront d'abord la condition humaine au coeur du monde, histoire et culture comprises (ce sera l'objet de la présente section), les tâches plus proprement théologiques ensuite (indiquées en postlude) qui me paraissent ici requises et possibles.

Mon premier plaidoyer visera à souligner et à valider les traits de contingence qui marquent notre condition. Contingence profonde, irréductible et potentiellement heureuse. Contingence à reconnaître sans reste et à laquelle faire face comme ce qui nous est donné, mais qui peut être tout à la fois le lieu et l'occasion d'un dépassement, interne et mystérieux, passant par désaisissement, ouverture, accueil renouvelé et en ce sens don.

La contingence est irréductible dès lors que toute remontée linéaire vers une origine première s'avère coupée, et dès lors qu'une hétérogénéité apparaît première et constitutive. Le cosmos comme tel n'est pas secrètement humain, mais incommensurable, énigmatique et disproportionné; l'humain n'a pas proprement à s'y résorber, même s'il doit certes y faire face, suspendre son jugement, se laisser interroger, s'étonner et d'une certaine manière en accueillir l'étrangeté. Parallèlement et en toute cohérence, on tient ici que ni le cosmos ni l'humain ne sont comme tels divins: s'ils ont certes à être reçus, c'est comme venant d'un autre et cristallisant en eux-mêmes tout à la fois une valeur propre, spécifique, bonne en ses limites mêmes, et un renvoi possible, indirect, à ce que, justement, ils ne sont pas, mais dont ils peuvent, au plus mais décisivement, témoigner.

Dire la contingence irréductible, c'est dire, pour l'humain, la précédence du monde: les choses sont là avant moi, comme elles seront d'ailleurs là après moi. Rigoureusement, on ne sait d'où elles viennent (mais seulement comment, de qui et pour qui je peux présentement les recevoir), ni, rigoureusement toujours, où elles vont. Même si mon destin y est d'une certaine manière lié, au sens où ma vocation propre ne saurait en faire l'économie, je ne fais pas nombre avec elles. D'ailleurs, bibliquement, le monde "passe», et il n'est pas promis à «résurrection».

Mais, irréductible, la contingence redouble en quelque sorte en ce que les choses justement, de toujours déjà là, pour moi, ne sont, pour moi toujours, pas données de manière vierge et pure, comme "au commencement». Elles sont dès le départ imbriquées dans une histoire et une culture, elles me viennent chargées de signes, positifs et négatifs. 
Elles sont orientées et constituent justement un cosmos ou un monde, non un ensemble neutre et sans ordre; et elles sont insérées ou portées par un ordre ambivalent d'amour et de refus: je puis, alternativement ou en partie simultanément, les accueillir ou les refuser, les investir comme lieu d'habitation ou les récuser dans un geste de révolte.

L'irréductibilité de la contingence et de l'imbrication indiquée ici va loin. Elle interdit un rapport à soi qui serait originaire et transparent, qu'il soit donné ou à restituer. Un ordre de médiations - multiples - sera au contraire requis et non dépassable. Même dans le rapport à soi-même. À fortiori dans le rapport aux choses et à Dieu ou à l'absolu.

Personnellement, c'est sur le fond d'une telle position que je crois devoir accorder une priorité à ce qu'on a pu appeler, en perspective phénoménologique notamment, le «monde de la vie» ${ }^{9}$ ou, chez Jean-Marc Ferry par exemple, l' «expérience» ${ }^{10}$. Lieu "originaire» tout en étant profondément historique et incarné. Aux antipodes d'un naturalisme neutralisant, intégrateur et résorbant finalement toute subjectivité; aux antipodes aussi d'un subjectivisme ou d'un idéalisme desserti des appartenances tant naturelles qu'historiques et culturelles. Refus donc de délier le sujet humain et le monde des choses, que ce soit pour faire fond sur l'un au détriment de l'autre - ils sont non isolables, sauf nostalgie secrète ou prométhéisme caché -; mais refus aussi, bien sûr, de tout dualisme, pouvant ouvrir sur des compositions diverses des éléments en cause. Encore une fois, pour l'homme, la complexité ou la coappartenance, de même que les histoires diverses qui la strient, sont originaires, constituent mon présent et demeurent ${ }^{11}$.

Mais complexité et coappartenance ne veulent pas nécessairement dire impossibilité de penser (donc de différencier), absence de mise en perspective et d'orientation, mise en échec de toute régulation. Le penser du monde, de l'humain et de l'absolu sera simplement réflexif, c'est-àdire second. Comme tel, il ne sera pas de l'ordre d'une connaissance ou d'un savoir, bien qu'il suppose des connaissances et des savoirs (limités et

9 Voir par exemple et en fonction du présent contexte de discussion, Maria VILLELA-PETIT, “Nature, monde et terre», dans De la nature, p. 255-281.

10 Les puissances de l'expérience. Paris, Cerf, 1991.

11 Il y a là des éléments à prendre en compte dans une réflexion sur ce qu'il en est du sujet, thématique centrale au coeur de la modernité comme de la "post-modernité», voir, non sans rapport avec certains éléments mis ici en évidence, Alain TOURAINE, Critique de la modernité. Paris, Fayard, 1992 (partie III). 
précis à chaque fois, même si leur extension peut mener très loin, comme c'est tout particulièrement le cas aujourd'hui) touchant les éléments du monde, leurs combinatoires et leurs utilisations possibles. Le penser sera articulé en tous points aux jeux de représentations et aux histoires qu'elles commandent dans le rapport de l'humain à lui-même, aux choses et à ses dieux. Il passera par une lecture de la culture et des mythes - traversée notamment et en outre d'une interrogation généalogique -, tant de leurs déploiements anthropologiques (notamment dans les registres du symbolique et du rituel qui sous-tendent nos habitations du monde) que de leur quête propre (jusqu'au rapport à l'absolu qui se tapit derrière leurs désirs ou leurs refus, leurs justifications intimes ou leurs utopies).

À propos d'une imbrication originaire de l'humain et des choses, j'ai évoqué un voisinage de la phénoménologie. Parlant d'un penser réflexif et second, différent d'un connaître, on peut évoquer maintenant une proximité à l'endroit de Kant. Mais il s'agit d'un Kant, on l'aura compris, dont tout moment "transcendantal» reste justement de bout en bout réflexif et ne saurait faire nombre avec les choses du monde. Il y est inscrit, mais on ne peut en parler et l'évoquer qu'à propos et en fonction d'effectivités lues et mises en perspective. La subjectivité humaine notamment ne se constitue qu'au travers d'une concrétude irréductiblement chargée de chair et d'histoire, dès lors à jamais particulière, aussi vrai que la donation même du monde apparaît elle aussi toujours incarnée et prise dans un ordre de figures historiques.

Le sujet est ici réponse, certes en responsabilité personnelle, donc en différence; mais dans la mesure même où il est réponse - ce qui veut dire aussi riposte -, il s'avère porteur de cela même à quoi il répond, par rapport à quoi il se pose et qu'il prend en charge. L'humain est réponse, au présent et en singularité - hors toute pure résorption dans les choses et le temps qui passe -, mais c'est au sens où il répond $d u$ monde, comme il répond d'ailleurs, en même temps, de son existence même et ainsi, indirectement, au monde, aux sollicitations et aux questions qu'il y déchiffre. Parce qu'il répond ainsi $d u$ monde, c'est aussi, pour une part, le monde même qui vient se dire au travers de l'humain, le monde qui, comme cosmos ou création, est plus ou autre que simple juxtaposition des choses. Le monde vient se dire là au travers du geste par lequel l'humain se pose dans les choses et le temps, dans la mesure même où l'humain ne se pose en vérité que via un accueil au plus intime de lui-même de ce qu'il n'est pas comme sujet mais qui s'est cristallisé en son corps, corps propre et matière. 
Plaider pour l'irréductibilité de la contingence - incontournable et seule porteuse de promesse pour un accomplissement vrai de l'humain va de pair avec un plaidoyer en faveur d'un Dieu qui ait franchement figure de transcendance (en rupture avec le donné) ou d'absolu (littéralement: ce qui est non lié). De même que le thème du monde comme contingence, celui de Dieu comme absolu répond au motif biblique et chrétien relatif à la création; et l'un comme l'autre me paraissent pouvoir heureusement prendre place au coeur de notre aujourd'hui le plus contemporain: être tout à la fois pertinent et susceptible de faire voir là des enjeux centraux et d'ouvrir, en contraste, des pistes fécondes.

Pour l'exprimer dans des termes qui ont partie liée avec des thématiques tout particulièrement évoquées au coeur même de la modernité - centralement chez Pascal ${ }^{12}$, en aval et à sa manière chez Kierkegaard, en amont tant dans un courant mystique qu'au sein de la théologie des grands Réformateurs, Calvin et Luther -, je dirais volontiers que Dieu est "caché". Non évident, du fait même de l'hétérogénéité, des discontinuités ou des différences foncières que j'ai ici évoquées tout au long. Un Dieu "caché», dont la confession est indirecte, passant par un débat avec un monde ouvert, énigmatique et inépuisable, un monde tout entier précédence et qui vaut comme parabole d'une précédence plus radicale.

Je le disais d'entrée, la modernité est née avec la conscience qu'un monde profondément unitaire prenait fin. Une précarité s'en dégageait; chacun se trouvait délogé de son "lieu». Une aventure y a pris naissance. Prométhéenne en sa forme dominante. Mais si l'humain se trouvait alors effectivement plus radicalement interpellé, la riposte pouvait être - et a aussi été parfois - différente. Non au gré d'un refus de la modernité, mais d'une autre manière d'en honorer le fait aussi bien que la quête sousjacente. Une contingence de départ peut être ici radicalisée, aussi vrai que le savoir et la maîtrise n'en viendront pas à bout, mais, subrepticement, l'escamotent plutôt; la transcendance peut s'en trouver également réaffirmée, voire retrouvée: rien dans le monde ou en l'homme n'a forme de divin ou ne vient directement de Dieu, sauf idolâtrie et idéologie, phénomènes ô combien modernes pour leur part!

On ne le sait que trop, le religieux et la théologie peuvent se trouver, en modernité, marginalisés. À la mesure même d'une affirmation de

12 Voir mon «Avant-propos: Port-Royal pris dans les jeux de la mémoire», dans Pour ou contre Sainte-Beuve: Le «Port-Royal». Genève, Labor et Fides, 1993, p. 5-11. 
l'homme à partir de lui-même, cet homme fragilisé, sommé de réagir et répondant par une sorte de fuite en avant. Mais la même dimension religieuse et le même ordre de réflexion proprement théologique peuvent aussi, en modernité, être relancés. Les institutions ecclésiales et l'adhésion à un Credo semblent en effet souvent vouées à la marginalité et apparaître déconnectées du réel (à moins d'être impétueusement réaffirmées dans des modalités fondamentalistes ou intégristes); mais les dieux peuvent se retrouver, non identifiés, déniés et en tout cas non régulés comme tels, au coeur de l'histoire et du rapport de l'humain au monde. L'histoire politique en a fourni des exemples en ces temps traversés de totalitarismes divers; l'histoire personnelle pourrait en fournir d'autres. Une réalité "sauvage» du religieux fait retour à l'heure d'une mort officielle de Dieu (dans l'ordre de la raison et des normativités publiques), où l'on peut voir à l'oeuvre des fonctionnements de frappe justement religieuse, anthropologiquement parlant, et où le théologien peut aussi déchiffrer la thématique d'un rapport à l'absolu qui, loin d'être conjurée ou dépassée, se donne au contraire libre cours ${ }^{13}$.

Soulignons-le, si l'humain peut ici apparaître comme étant aux prises avec une instance religieuse et animé par une quête d'absolu, c'est en toute sécularité ou en toute profanité; c'est au coeur du monde pris dans toute sa consistance et son déploiement propre. L'instance religieuse comme la quête, le refus ou la dénégation de l'absolu ne se montrent et ne se disent qu'à titre indirect, même si c'est au gré d'une mise en perspective, d'une interrogation et d'une problématique spécifiques. C'est que le religieux et la théologie n'ont pas affaire en dernière instance à des domaines propres, séparés ${ }^{14}$; tel était d'ailleurs, justement, l'un des enseignements majeurs de la tradition biblique et chrétienne relative à la création: ce qui est en cause, c'est le réel et le rapport qu'y noue l'humain comme tel; et c'est en fonction de Dieu comme tel qu'on confesse à ce propos un aveu et une promesse, aussi vrai que la question dernière porte, indirectement, sur mon rapport originaire à ma propre existence et à ce qui m'est donné, non à un énoncé particulier, à une morale spécifiée, au culte ou à la reconnaissance d'un Dieu parmi d'autres et qui serait, lui, le vrai...

13 Sur ce point, voir mon ouvrage L'excès du croire. Paris, Desclée de Brouwer, 1990, chapitre 6.

14 Même si la lecture d'une tradition particulière peut se révéler ici exemplaire et apparaître, à sa manière et en son ordre, incontournable. Sur ce point, voir ma contribution «Apories et pertinence de la métaphysique», BullLitEccl 94/3 (1993) 235. 254. 
Pour une position de l'humain entre contingence, donnée, et absolu, hors monde; tel était ici le propos. La perspective exige, précisons-le encore, une valorisation du moment de l'expression; dans la mesure même où le réel est irrévocablement renvoyé à sa contingence et Dieu à sa transcendance ou à son absolu. Moment de l'expression comme fait profondément humain; moment qui est à la fois celui des représentations (elles sont requises en ce que le savoir ne vient pas à bout du monde ni de mon existence) et celui d'une exteriorisation où s'affirme une quête (extériorisation elle aussi requise, du fait de mon inadéquation au monde et à l'être). Les énoncés, les textes et les symboles se tiennent ici en première ligne, tant avec leurs configurations propres, situées, qu'avec ce qui les traverse et permet de les lire et de les recevoir comme témoignages. Rappelons qu'en économie chrétienne bien comprise, si ni Dieu ni le monde ne sont comme tels donnés en transparence ou en ligne directe, on ne renvoie pas non plus à une médiation en forme d'intermédiaire (la révélation n'est pas celle du Christ comme "nature» propre ${ }^{15}$ ) ni à un moment d'institution auto-assurée ou accréditée par autorisation strictement externe (le passage par le texte justement est incontournable et au reste seul canonique $\left.{ }^{16}\right)$. En d'autres termes, c'est indiquer là qu'il n'y a pas de confession authentique de Dieu hors d'un moment proprement esthétique (moment qui n'est pas levable, à la mesure même de l'énigme du monde, également non levable, ou qu'on ne lève que dans le "péché»: la propension diabolique à tout ramener secrètement à soi). C'est pourquoi, en doctrine, le Christ est tout à la fois contemplé et présenté comme figure où se joue un destin qui, en toute radicalité de terme, dépasse sa personne, son vouloir et son savoir (le procès est de dimensions cosmiques ${ }^{17}$ : y sont engagées la vie et la mort, la malédiction et la bénédiction, et on dit le Christ «livré» et "fait péché»).

15 Doctrinalement, on ne connaît que la nature humaine et la nature divine, ainsi qu'une mise en rapport des deux qui peut être reçue comme révélatrice de leur identité à chacune, tant dans leur vérité profonde que dans leur dévoilement effectif.

16 À moins d'idolâtrer Jésus à force de le vouloir Christ..., cela dit en toute fidélité traditionnelle, la tradition étant comprise ici dans ce qu'elle a de plus central, de meilleur et de plus fort.

17 Voir à ce sujet mon ouvrage Le Christ de Calvin. Paris, Desclée, 1990 (en particulier chapitre 5) et «Nécessité du moment esthétique en théologie et pour la théologie» (à paraître, Actes du colloque Tillich, Montpellier 1993). 


\section{Postlude}

Je le disais d'entrée, la modernité a notamment eu pour effet de délier les savoirs du monde des questions touchant le croire. La théologie chrétienne s'est dès lors souvent repliée (pas toujours!), en toute conscience ou non, sur des réalités de fait sotériologiques ${ }^{18}$ et ecclésiologiques, tendant dans la foulée à réduire sa tâche propre au seul intellectus fidei ${ }^{19}$.

Je plaide pour une remise en honneur du thème de la création. $\grave{A}$ cause de sa richesse propre bien sûr. Mais aussi parce que je crois nécessaire et légitime, urgent et bénéfique, d'articuler délibérément les données de la foi ainsi que l'interrogation théologique aux réalités du monde de tous, en universalité donc. Un tel souci renoue d'ailleurs avec la tradition la plus classique du christianisme, qui a certes pu mener à des prétentions formellement illégitimes (qu'il faut dénoncer!), mais dont, correctement située, l'exigence doit demeurer: l'horizon de la théologie ne saurait être que le monde comme tel et la question que celle de Dieu comme tel. La vérité proposée doit l'être comme vérité de tous et doit être pensée ainsi (sans quoi elle n'est pas vérité), même si elle n'est pas la seule, si elle est singulièrement enracinée et assumée, si elle est donc en dialogue voire en confrontation et en interaction avec d'autres.

Précisons à ce propos, sans pouvoir le développer ici, qu'est dès lors requise, d'abord, une réflexion sur les instances différentes qui strient la vie humaine, en société moderne tout spécialement; dans ce cadre, on devra notamment se mettre au clair quant à une "théorie du religieux": sa fonction propre et son articulation à d'autres instances, sur le fond d'un horizon universel. Sera également requis, ensuite, un «dialogue interreligieux» touchant la manière même de donner corps à cette instance ou à ce moment religieux, un dialogue qui ne peut, à mon sens, qu'être celui d'une confrontation à partir de positivités historiques effectives, donc particulières, validant leurs différences, mais montrant aussi comment elles assument à chaque fois, différemment, des réalités plus larges,

18 Des historiens ou des exégètes ont d'ailleurs proposé de comprendre le thème biblique de la création comme pure extension ou radicalisation d'une confession de salut (ou de «libération», en l'occurrence l'exode hors d'Égypte, voir par exemple Gerhard von RAD).

19 Exemplairement, en protestantisme, dans la "théologie dialectique" (dès les années 20 de ce siècle, avec les noms de Karl BARTH et Rudolf BULTMANN), mais non sans parallèles, plus récents, dans le catholicisme. 
globalement anthropologiques si l'on veut ${ }^{20}$. Là aussi l'horizon sera universel, mais là encore non en vue de réduire les différences et les particularités, mais bien plutôt afin de les situer, de les recevoir et d'une certaine manière de les approfondir, via détour et décentrement. Sera requis enfin un va-et-vient plus interne au travail théologique entre la lecture des moments positifs d'une tradition donnée, en l'occurrence chrétienne (moments historiques, institutionnels et doctrinaux, bibliques et ecclésiaux), et la reprise réflexive de ce qui est à chaque fois en jeu quant à l'homme et à sa position dans le monde, quant au monde lui-même ou à l'être, quant à l'absolu confessée ${ }^{21}$.

En tout cela, il n'est pas question de nier la différence entre savoirs du monde et ordre de croyance, mais bien de les articuler, dans un jeu aux plans et aux interférences complexes, à distinguer soigneusement à chaque fois, sachant en outre qu'une telle articulation aura bien sûr des effets en retour sur chacun des moments et des énoncés en jeu, propositions de foi comprises. Pour ma part, reprenant ici concrètement le thème biblique et chrétien touchant la création, je n'ai pas, me semble-t-il, nié la différence entre savoirs et croire. Mais je me suis bel et bien efforcé d'empêcher que cette différence ne mène à consacrer une séparation de deux domaines. La différence doit au contraire être travaillée, de l'intérieur; elle peut être ainsi dépassée en tant que partition et retrouvée dans ce qu'elle peut avoir de fécond, de part et d'autre et pour chacun.

20 Sur le type de dialogue ici envisagé, je renvoie à mon ouvrage La subversion de l'Esprit. Genève, Labor et Fides, 1993, p.189 ss.

21 C'est au vu de l'ensemble de ces tâches (tout particulièrement des deux premières) et de l'organon de travail qui leur est lié que s'affirme à mon sens aujourd'hui une nouvelle pertinence de la lecture de Ernst Troeltsch. 